\title{
ENTEROBACTERIAS RESISTENTES A CARBAPENEMICOS EN EL HOSPITAL MARIO CATARINO RIVAS DURANTE EL PRIMER SEMESTRE DEL 2016
}

\section{Carbapenem Resistant Enterobacteriaceae at Hospital Nacional Mario Catarino Rivas during the First Semester of $\mathbf{2 0 1 6}$}

*José Edgardo Laureano Alvarado, **Stephanie Guadalupe Pineda López.

\section{RESUMEN}

Introducción: El notable aumento de las infecciones producidas por enterobacterias resistentes a carbapenémicos (por sus siglas en ingles CRE) y su impacto negativo en las temáticas de salud, han generado preocupación y han sido motivación para investigar la epidemiologia propia. Objetivo: Determinar la prevalencia de las CRE y los sitios de aislamiento más frecuentes en el Hospital Nacional Mario Catarino Rivas (HNMCR) desde el 1ero de enero al 30 de junio del 2016. Metodología: El estudio fue de tipo descriptivo, transversal, se obtuvo los datos de los libros de registros diarios del departamento de microbiología, según la definición recomendada por la Centro para el Control y Prevención de Enfermedades (CDC) utilizando parámetros de sensibilidad de la Clinical Laboratory Standards Institute (CLSI) M100 S24 Resultados: Se reportó el aislamiento de 696 enterobacterias de las cuales 71(10.2\%), cumplían la definición de la CDC para CRE; Klebsiella pneumoniae 21 (29.58\%), Enterobacter aglomerans 20 $(28.17 \%)$ y Escherichia coli 12 (16.9\%). Las salas de emergencia de cirugía, cirugía de hombres, cirugía de mujeres, medicina interna de mujeres y lactantes presentaban cada una $4(5.63 \%)$ casos reportados, haciendo un total de $27.65 \%$. En abril se reportaron 15 CRE $(21.13 \%)$, aislándose principalmente en urocultivos 26 (36.6\%). Conclusiones: Klebsiella pneumoniae fue la CRE más prevalente en HNMCR. Abril fue el mes con más reportes de CRE y el urocultivo fue el sitio de aislamiento más frecuentemente reportado.

\footnotetext{
*Médico en Servicio Social de la Escuela Universitaria de Ciencias de la Salud. EUCS-UNAHVS

${ }^{* *}$ Estudiante de $5^{\circ}$ de la carrera de medicina EUCS-UNAHVS

Dirigir correspondencia a: jelaureano@hotmail.com

Recibido: 09 de septiembre 2016 Aprobado: 15 de diciembre 2016
}

\section{PALABRAS CLAVE}

Diagnóstico, enterobacteriaceae, epidemiología y microbiología.

\begin{abstract}
Background: The noticeable increase of carbapenem resistant enterobacteriaceae (CRE) and its negative impact in health issues have generated worries and have motivated a national research of our epidemiology. Objective: the main aim of this study was to obtain the prevalence of CRE and the most frequent isolates sites at Hospital Nacional Mario Catarino Rivas (HNMCR), done from January 1st up to June 30th of 2016. Methodology: descriptive, transversal, prospective study. Data was obtained from the daily microbiological registry of the Department of Microbiology at HNMCR. CRE was defined according Center for Disease Control and Prevention (CDC) standards using the sensibility parameters of the Clinical Laboratory Standards Institute (CLSI) M100 S24. Results: 696 enterobacteriaceae were reported of which $71(10.2 \%)$ met the CDC phenotypic definition of which Klebsiella pneumoniae reported 21 (29.58\%), Enterobacter aglomerans 20 $(28.17 \%)$ and Escherichia coli 12 (16.9\%). CRE were isolated in $4(5.6 \%)$ patients in each of the following areas in the hospital: surgery emergency room, womens surgery ward, infants ward and women internal medicine ward, thus representing $27.65 \% .15(21.13 \%)$ CRE were isolated in April and 26 (36.6\%) were isolated in urine cultures. Conclusions: Klebsiella pneumoniae was the most prevalent CRE in HNMCR. April presented the highest CRE reports. The isolates from urine cultures were the most frequently reported.
\end{abstract}

\section{KEYWORDS}

Diagnostic, enterobacteriaceae, epidemiology, microbiology. 


\section{INTRODUCCIÓN}

La prevalencia de las enterobacterias productoras de carbapenemasas han experimentado en los últimos años un aumento a nivel global, lo que se hace indispensable adoptar medidas para controlar esta epidemia. Desde su primer asilamiento en 1997 con la cepa Nueva Delhi, se ha aislado principalmente $\mathrm{K}$. pneumoniae como la principal enterobacteria productora de carbapenemasas siendo también el mecanismo OXA-48 el principal mecanismo molecular de resistencia bacteriana con mayor prevalencia en el sur de Europa, Asia y América del Sur. ${ }^{(1-3)}$

Ante la amenaza creciente de estas infecciones que en su mayor parte son nosocomiales se han realizado múltiples trabajos de investigación con el fin de detectar oportunamente, describir características epidemiológicas, aislar genotipos, y establecer medidas de control. ${ }^{(3-5)}$

Se han identificado enzimas, a las que se le atribuye mayor mecanismo de resistencia denominadas genéricamente carbapenemasas, pertenecen en su mayoría a 3 clases diferentes, según la clasificación molecular de Ambler. ${ }^{(6)}$

a) Clase $A$, principalmente enzimas del tipo $\mathrm{KPC}$.

b) Clase $B$ métalo--lactamasas (MBL) dependientes de zinc, principalmente enzimas del tipo VIM, IMP y NDM.

c) Clase D o serin-carbapenemasas (principalmente OXA-48).

Es muy importante realizar un cribaje periódicamente para detectar potenciales enterobacterias resistentes a carbapenémicos y así tomar medidas oportunas para el control de los casos reportados. ${ }^{(7)}$ La CDC (Center for Disease Control and Prevention) recomienda fuertemente la realización de pruebas fenotípicas (test de Hodge modificado, microdilución con ácido borónico) de enterobacterias resistentes a carbapenémicos en las unidades de salud y solamente con fines epidemiológicos recomienda confirmarlos a través de pruebas genotípicas (PCR). ${ }^{(8,9)}$ A nivel regional los estudios publicados revelan que la cepa KPC es la enterobacteria resistente a carbapenémicos con mayor prevalencia en el istmo latinoamericano seguida muy de cerca de la cepa Métalo B lactamasa de Nueva Delhi el cual presenta brotes sobre todo en los meses de abril a agosto. ${ }^{(10-12)}$ Las CRE constituyen una morbilidad y mortalidad muy importante en lo que respecta a las infecciones nosocomiales registrándose hasta un $50 \%$ de mortalidad en los pacientes infectados con CRE y representan el segundo lugar solamente superado por P. aeruginosa. ${ }^{(13)}$ El tratamiento de las infecciones producidas por enterobacterias resistentes a los carbapenémicos constituye un gran dilema porque resultan ser resistentes a múltiples fármacos. ${ }^{(14)}$ Según Latania (2015), en un estudio retrospectivo, la CRE es más frecuente en niños de 14 años, el género Enterobacter predominó en un 37\% aislándose sobre todo en urocultivos en un $77 \%$ de los casos, CRE confirmados donde se ha observado un incremento en 14 años de 0.47 a un $3.3 \%$ y encontrándose mayor prevalencia en la unidad de cuidados intensivos con un incremento de 0.5 a $4.5 \%$. ${ }^{(15-17)}$

Dado la importancia, es indispensable realizar el primer estudio de prevalencia de enterobacterias productoras de carbapenemasas a nivel nacional, ya que hasta la fecha no poseemos información epidemiológica de esta índole. A nivel Centroamericano en Noviembre del 2011, el Centro Nacional de Enlace para el Reglamento Sanitario Internacional en Guatemala emitio una alerta epidemiológica por el aislamiento de cepas Klebsiella pneumoniae multirresistente por carbapenemasas tipo Nueva Delhi metalobetalactamasa. Las dos cepas ailada de $K$. pneumoniae procedían de 2 hospitales de referencia nacional situadas en la ciudad de Guatemala. ${ }^{(18)}$

Según Lubert et al (2015) y Lefevbre et al (2015) los programas de vigilancia epidemiológica deben realizarse durante 2 a 3 veces en el año e indagar sobre la procedencia de los casos confirmados ya que en la actualidad constituye no solamente un problema intrahospitalario sino que se han reportado algunos casos a nivel comunitario. ${ }^{(19-21)}$ 
El objetivo principal de la investigación fue determinar la prevalencia de enterobacterias productoras de carbapenemasas en el HNMCR durante el 1 de enero al 30 de junio del 2016.

\section{METODOLOGÍA}

Se realizó un estudio descriptivo, transversal, durante el 1 de enero al 30 de junio del 2016 en el laboratorio del HNMCR. Universo: Se registraron un total de 696 enterobacterias de las cuales 71 cumplieron la definición propuesta por la CDC para CRE utilizando el CLSI M100 S24, procedentes de las diferentes salas del HNMCR. Criterios de inclusión: Enterobacterias en las cuales se midió la susceptibilidad con un carbapenem (excepto ertapenem). Enterobacterias reportadas en los registros microbiológicos de cultivo durante el periodo descrito y aquellas reportadas en los registros microbiológicos de cultivo procedentes de las salas del HNMCR. Criterios de Exclusión: Enterobacterias en las cuales no se midió la susceptibilidad con un carbapenem. Enterobacterias reportadas en los registros microbiológicos de cultivo fuera del periodo comprendido entre el 1 de enero al 30 de Junio. $Y$ aquellas reportadas en los registros microbiológicos de cultivo no procedentes de las salas del HNMCR.

Procedimiento: Se realizó una revisión sistemática y minuciosa de los registros microbiológicos de cultivos del Hospital Mario Catarino Rivas, tomando en cuenta los criterios de inclusión y exclusión anteriormente citados y depositándolos en una tabla mensual de recolección de datos y posteriormente en 3 tablas matrices. Los procedimientos de este departamento cumplen con los criterios de sensibilidad de la CLSI (Clinical and Laboratory Standards Institute) M100 S24 a través del método de difusión en disco de Kirby Bauer para enterobacterias resistentes a carbapenémicos: "resistencia comprobada a cualquier carbapenémico (excepto ertapenem) más resistencia a toda cefalosporina de 3ra generación. En el estudio actual solo se utilizó imipenem como carbapenemico.
Análisis estadístico: Se utilizaron las herramientas estadísticas de Microsoft Excel 2013 para la cuantificación de prevalencias, medidas de frecuencia y porcentajes de los datos obtenidos, representándolos en tablas y gráficos.

Aspectos Éticos: Este es un estudio categoría 1. En ningún momento involucro el contacto personal con los pacientes hospitalizados, no se divulgaron ninguno de los datos personales de los sujetos a los que se les realizaron los diversos cultivos. Se obtuvo acceso a los libros de registro microbiológicos del departamento de laboratorio del Hospital Mario Catarino Rivas previo consentimiento de las autoridades del departamento.

Financiamiento: Dicho estudio no fue subsidiado económicamente más que por los propios medios de los autores.

\section{RESULTADOS}

Se reportó el aislamiento de 696 enterobacterias de las cuales 71 (10.2\%), cumplían la definición de la CDC para CRE; Klebsiella pneumoniae representó $21(29.58 \%)$ y Enterobacter $20(28.17 \%)$. (Ver tabla No. 1.)

\section{Tabla No. 1: Enterobacterias Resistentes a Carbapenémicos}

\begin{tabular}{|c|c|c|}
\hline $\begin{array}{c}\text { Genero } \\
\text { Enterobacteria }\end{array}$ & $\begin{array}{c}\text { No. de } \\
\text { Enterobacterias }\end{array}$ & Porcentaje \\
\hline Klebsiella & 21 & $29.58 \%$ \\
\hline Enterobacter & 20 & $28.17 \%$ \\
\hline Escherichia & 12 & $16.90 \%$ \\
\hline Serratia & 8 & $11.26 \%$ \\
\hline Proteus & 7 & $9.86 \%$ \\
\hline Citrobacter & 3 & $4.23 \%$ \\
\hline Total & 71 & $100.00 \%$ \\
\hline
\end{tabular}

Fuente: Libros de Registro Diario de Cultivos del Departamento de Microbiología del HMCR

Las salas de emergencia de cirugía, cirugía de hombres, cirugía de mujeres, medicina interna de mujeres y lactantes presentaban cada una $4(5.63 \%)$ casos reportados y $6(8.45 \%)$ en salas desconocidas (otras salas). (Ver tabla No. 2.) 
Tabla No. 2: Distribución de las CRE por sala

\begin{tabular}{|l|c|c|}
\hline Sala & $\begin{array}{c}\text { No. Entero- } \\
\text { bacteria }\end{array}$ & $\begin{array}{c}\text { Porcen- } \\
\text { taje }\end{array}$ \\
\hline Desconocido & 6 & $8.45 \%$ \\
\hline Emergencia de cirugía & 4 & $5.63 \%$ \\
\hline Cirugía de hombre & 4 & $5.63 \%$ \\
\hline Cirugía de mujer & 4 & $5.63 \%$ \\
\hline Lactante & 4 & $5.63 \%$ \\
\hline Medicina de mujer & 4 & $5.63 \%$ \\
\hline Emergencia de pediatría & 3 & $4.23 \%$ \\
\hline Ginecología & 3 & $4.23 \%$ \\
\hline Neurocirugía Pediátrica & 3 & $4.23 \%$ \\
\hline Ortopedia de hombres & 3 & $4.23 \%$ \\
\hline Ortopedia de mujeres & 3 & $4.23 \%$ \\
\hline Medicina de hombres & 3 & $4.23 \%$ \\
\hline Medicina materno fetal & 3 & $4.23 \%$ \\
\hline Nefrología de hombre & 3 & $4.23 \%$ \\
\hline Labor y parto & 3 & $4.23 \%$ \\
\hline Neonatos & 3 & $4.23 \%$ \\
\hline Consulta externa & 3 & $4.23 \%$ \\
\hline Emergencia medicina interna & 2 & $2.81 \%$ \\
\hline Unidad de cuidados intensivos & 2 & $2.81 \%$ \\
\hline Adolescentes pediatría & 2 & $2.81 \%$ \\
\hline Recuperación & 2 & $2.81 \%$ \\
\hline Medicina pediátrica & 1 & $1.41 \%$ \\
\hline Centro de atención integral & 1 & $1.41 \%$ \\
\hline Cirugía vascular & 71 & $1.41 \%$ \\
\hline Hematooncología & $1.41 \%$ \\
\hline Total & 3 & $100 \%$ \\
\hline & 3 & 3 \\
\hline
\end{tabular}

Fuente: Libros de Registro Diario de Cultivos del Departamento de Microbiología del HMCR

Se encontró que abril fue el mes donde más casos de CRE se reportaron 15 (21.13\%), seguido de febrero y marzo $13(18.31 \%)$ correspondiente a cada uno. (Ver gráfico No.1.)

\section{Gráfico No. 1: No. de CRE aisladas por mes}

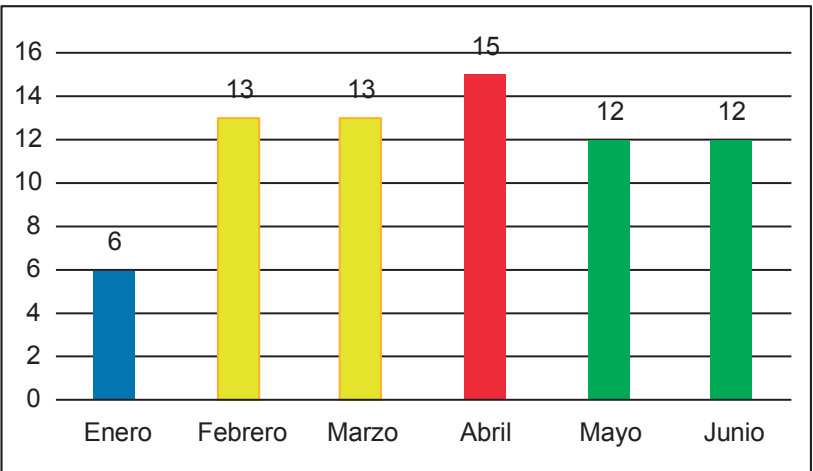

Fuente: Libros de Registro Diario de Cultivos del Departamento de Microbiología del HMCR
En cuanto a los sitios de aislamiento de CRE, nótese que el urocultivo fue más frecuente con 26 casos (36.6\%). (Ver gráfico No. 2.)

\section{Gráfico No. 2: Distribución de CRE por Sitio de Aislamiento}

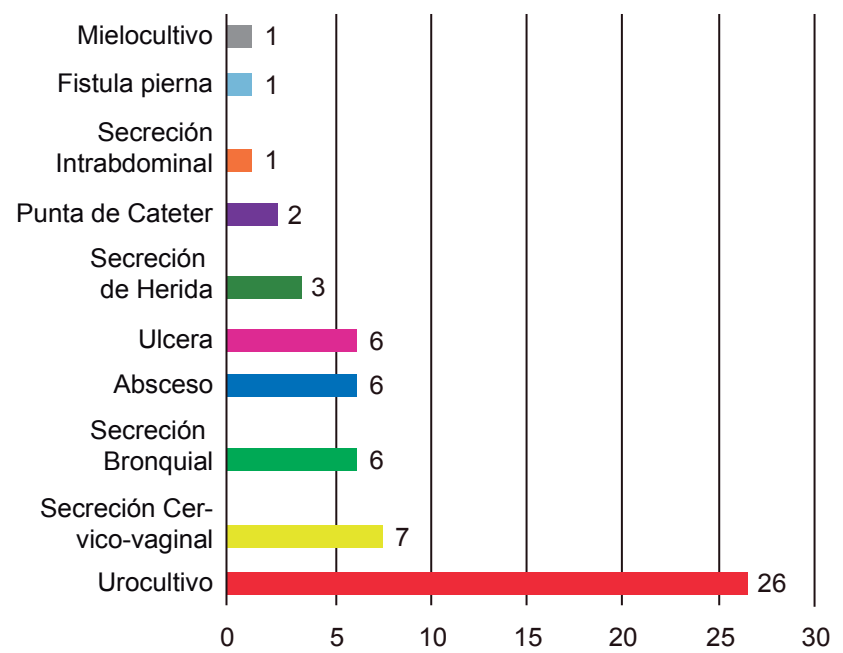

Fuente: Libros de Registro Diario de Cultivos del Departamento de Microbiología del HMCR

\section{DISCUSIÓN}

Las infecciones nosocomiales producidas por las enterobacterias representan una importante morbimortalidad, teniendo una amplia capacidad de diseminación, solamente superadas por las infecciones ocasionadas por el género de Pseudomonas. Se identificaron 71 casos de CRE que cumplieron la definición fenotípica propuesta por la CDC de las 696 enterobacterias reportadas presentando una prevalencia general de CRE del $10.2 \%$. Los cuales fueron distribuidos de la siguiente manera en orden descendente: Klebsiella pneumoniae 21 casos, Enterobacter aglomerans 20 casos y Escherichia coli 12 casos que representan el $29.58 \%$, $28.17 \%$ y $16.90 \%$ respectivamente. K. pneumoniae se perfila como la CRE más frecuentemente reportada. No obstante, debemos de recordar que la definición utilizada pudo haber identificado cierto porcentaje de falsos positivos y esto es muy probable ya que no realizamos microdilución en caldo ni pruebas fenotípicas (eg. Test modificado de Hodge) ni genotípicas para corroborar. Los resultados del programa nacional de vigilancia epidemiológica de los Estados Unidos en el 2015 reve- 
Iaron que la CRE más prevalente continúa siendo K. pneumoniae con una prevalencia del $49 \%$ y el mecanismo más frecuentemente reportado es el de Klebsiella pneumoniae productor de carbapenemasas siendo la cepa Nueva Delhi la prevalente. ${ }^{(22)}$ No contamos con información procedente de estudios nacionales ya que no se encontraron publicaciones en los buscadores virtuales (PubMed, BVS).

Las salas en estudio mostraron un resultado poco esperado ya que se desconoce el origen de las salas en un $8.45 \%$ lo cual nos deja la lección de fortalecer el registro microbiológico diario mediante el llenado completo de los datos a la hora de solicitar cultivos. Sin embargo en las salas de mayor prevalencia de CRE fueron emergencia de cirugía, cirugía de hombres y mujeres, medicina interna de mujeres y lactantes cada una presentaba $4(5.63 \%)$ casos reportados, conformando un total de $27.65 \%$. No se reportaron casos procedentes de la unidad de cuidados intensivos pediátricos (UCIP), sino que estos provinieron de la sala de cuidados intensivos de adultos, donde se reportó 2 casos de CRE representando (2.81\%). A diferencia del estudio presentado por Cuervo y colaboradores (2014), la sala donde se presentó mayor incidencia corresponde a la UCIP con un aumento de 0.02 a $4 \%$ en un lapso de 4 años. ${ }^{(23,24)}$

Es también interesante mencionar que el mes donde más se reportaron CRE correspondió a abril donde se reportaron 15 casos lo que representa el $21.13 \%$ de total de casos de CRE reportado, información similar reporta la CDC en los EEUU con el inicio del período de reporte de mayores casos de CRE en el año siendo más frecuente durante los meses de abril y agosto reportando un $30 \%$ de los casos. ${ }^{(25)}$ Se deberán realizar estudios analíticos para investigar los factores asociados a la incidencia alta durante estos meses, que según la CDC corresponden a hospitalizaciones prolongadas, infecciones cruzadas, uso de dispositivos invasivos, pacientes con inmunosupresión y uso de antibioticoterapia extensa. (Biehle et al, 2015). ${ }^{(26)}$

Se evidenció a su vez que el sitio donde más frecuentemente se aislaron CRE correspondió a los urocultivos 26 casos que representa $(36.6 \%)$, hallazgos similares han sido reportados por el estudio epidemiológicos realizado por Yamal W, Albert J, Vincent. en 7 comunidades urbanizadas de los EUA durante el 2015 , donde se reportaron $10(47.6 \%)$ casos de CRE aislados en urocultivos. ${ }^{(27)}$

Dificultades, Limitaciones y sesgos: como investigadores se nos dificulto acceder a los registros en horas laborales de mayor afluencia. Otra de las limitantes encontradas fue que no en todas las enterobacterias aisladas y reportadas se realizaron pruebas de sensibilidad con carbapenémicos por recursos económicos limitados del hospital. Razón por la cual los valores encontrados podrían ser aún mayores que los actualmente reportados.

Conclusiones: La enterobacteria resistente a carbapenémicos más frecuente aislada corresponde a Klebsiella pneumoniae. Las salas con mayores casos reportados correspondieron a: emergencias de cirugía, cirugía de hombres, cirugía de mujeres, medicina interna de mujeres y lactantes en igual magnitud. Abril fue el mes con mayor incidencia reportada y el urocultivo fue el sitio de mayor aislamiento de CRE.

Recomendaciones: Es de vital importancia fortalecer las medidas de bioseguridad a la hora de detectar los casos de CRE mediante el aislamiento del o los casos reportados, implementar métodos de barrera descartables a la hora de interactuar con los sujetos confirmados con una CRE y desinfectar las unidades ocupadas por los casos confirmados con posterior toma de muestras dada la alta posibilidad de resistencia principalmente de cepas de K. Pneumoniae. Con el fin de establecer comparaciones con las cepas y características poblacionales de los casos registrados e identificar potencialmente mecanismos clonales se deberían tomar muestras fenotípicamente confirmadas de las unidades de salud con más afluencia del país durante un período de 1 año para posteriormente realizar pruebas fenotípicas y dilucidar los mecanismos genotípicos subyacentes. 


\section{Conflictos de Intereses}

No se han establecido conflictos de intereses entre los autores.

\section{Agradecimiento}

Agradecemos al personal de laboratorio que colaboró facilitando los registros microbiológicos de cultivos del HMCR. A nuestra asesora técnica la Dra. Sagrario Calderón y a los siguientes investigadores participantes que colaboraron en la recolección de datos: Krisia Banegas, Allan Santos, Velia Rivera.

\section{REFERENCIAS BIBLIOGRÁFICAS}

1. Garza-Ramos U, Barrios H, Reyna-Flores $F$, Sánchez-Pérez A, Tamayo-Legorreta E, Ibarra-Pacheco A, et al. Characteristics of KPC-2-producing Klebsiella pneumoniae (ST258) clinical isolates from outbreaks in 2 Mexican medical centers. Diag Microbiol Infec Dis. [Internet] 2014 [citado el 5 de marzo 2016]; 79(4): 483-485. Disponible en: http://

ac.els-cdn.com/S0732889314002028/ 1-s2.0-S0732889314002028-main.pdf? $\mathrm{tid}=5 \mathrm{acfb} 068-$ eafd-11e6-b37b-00000 aacb35d\&acdnat $=1486228635$ d15ce87 b2cbd8030 dd9b084d5d784d9c.

2. Guh AY, Bulens SM, Mu Y, Jacob JT, Reno $\mathrm{J}$, Scott J, et al. Epidemiology of Carbapenem Resistant Enterobacteriaceae in 7 US communities, 2012-2013. JAMA [Internet] 2016 [citado el 7 Julio del 2016]; 314(14): 1479-1487.

Disponible en: http:// jamanetwork.com/ journals/jama/fullarti cle/2450329.

3. Lee BY, Bartsch SM, Wong KF, McKinnell JA, Slayton B, Miller LG, et al. The Potential Trajectory of Carbapenem-Resistant Enterobacteriaceae, an Emerging Threat to Health-Care Facilities, and the Impact of the Centers for Disease Control and Prevention Toolkit. Am J Epidemiol. [Internet] 2016 [citado el 7 agosto del 2016]; 183(5):471-479. Disponible en: https:// www.ncbi.nlm.nih.gov/pmc/articles/ PMC4772438/pdf/kwv299.pdf.

4. Padilha Tavares C, Silva Pereira P, de Andrade Marques E, Faria JrC, Araujo Herkenhoff de Souza MP, de Almeida R,
Morais Alves CF, et al. Molecular epidemiology of KPC-2-producing enterobacteriaceae (non-kilebsiella pneumoniae) isolated from Brazil. Diagn Microbiol Infect Dis. [Internet] 2015 [citado 4 Julio del 2016]; 82(1):326-330. Disponible en: http://www.dmidjournal.com/article/ S0732-8893(15)00122-4/pdf.

5. Tzouvelekis LS, Markogiannakis A, Piperaki E, Souli M, Daikos GL. Treating infections caused by carbapenemase-producing enterobacteriaceae. Clin Microbiol Infect. [Internet] 2014 [citado el 5 de Mayo del 2016]; 20(9):862-872. Disponible en: http:// www.clinicalmicrobiologyandinfection. com/article/S1198-743X(14) 65090-4/pdf.

6. Nordman P, Nass T, Poirel L. Global spread of cabapenemase-producing enterobacteriaceae. Emerg Infec Dis. [Internet] 2012 [citado el 22 junio del 2016];17(10);1792-1798.

Disponible en: https://wwwnc.cdc.gov/ei d/article/17/10/pdfs/11-0655.pdf.

7. Oteo J, Calvo E, Rodríguez Baño J, Oliver A, Homero A, Ruíz-Garbajosa P. [The treat of the carbapenemase-producing enterobacteriaceae in Spain: positionig report of the SEIMC study groups GEIH and GEMARA]. Enferm Infecc y Microbiol Clín. [Internet] 2014 [citado el 7 de octubre del 2016]; 32(10):666-670 Disponible en: http://ac.els-cdn.com/S0213005X140 00809/1-s2.0-S0213005X14000809-main. pdf? tid=bc61a120-eb15-11e6-b351-000 00aacb362\&acdnat=1486239107_143 cd0d68ee8b3bd01b5c78f5bab3797. 
8. Chea N, Bulens S, Konqp-Tran T, Lynfield $\mathrm{R}$, Shaw KM, Vagnone PS, et al. Improved phenotype-based definition for lidentifying carbapenemase producers among carbapenem-resistantenterobacteriaceae. Emerg Infect Dis. [Internet] 2015 [citado el 8 de septiembre del 2016]; 21(9):1611-6. Disponible en: https://www. ncbi.nlm.nih.gov/pmc/articles/PMC4550 143/pdf/15-0198.pdf.

9. Rodríguez EC, Saavedra SY, Leal AL, Alvares C, Olarte N, Valderrama A, et al. Diseminación de Klebsiella pneumoniae productoras de KPC-3 en hospitales de Bogotá durante un periodo de tres años. Biomédica [Internet]. 2016 [citado 01 Febrero 2016]; 34 (suppl 1): 224-231. Disponible en: http://www.scielo.org.co/ pdf/bio/v34s1/v34s1a25.pdf.

10. Mlynarcik P, Roderova M, Kolar M. Primer Evaluation for PCR and its Application for Detection of Carbapenemases in Enterobacteriaceae. Jundishapur J Microbiol. [Internet] 2016 [citado 23 de enero del 2016]; 9(1):e29314. Disponible en: https:// www.ncbi.nlm.nih.gov/pmc/articles/ PMC4834133/pdf/jjm-09-01-293 14.pdf.

11. CDC. Guidance for control of infections with carbapenem-resistant or carbapenemase- producing enterobacteriaceae in acute care facilities. MMWR Morb Mortal Wkly Rep. [Internet]. 2013 [citado 23 de enero del 2016]; 58(10):256-260.

Disponible en: https://www.cdc.gov/mm wr/pre view/mmwrhtml/mm5810a4.htm.

12. Xu Y, Gu B, Huang M, Lui H, Xu T, Wang T. Epidemiology of carbapenem resistant Enterobacteriaceae (CRE) during 20002012. J Thorac Dis. 2016 noviembre; 7(3): 376-385. Disponible en: https://www.ncbi. nlm.nih.gov/pmc/articles/PMC4387446/.

13. Pecora ND, Li N, Allard M, Li C, Albano E, Delaney $M$, et al. Genomically informed surveillance for carbapenem-resistant enterobacteriaceae in a health care system.
MBio.[Internet]. 2015 [citado el 10 agosto del 2016]; 6(4):1-11. Disponible en https:// www.ncbi.nlm.nih.gov/pmc/articles/ PMC4551976/pdf/mBio.01030-15.pdf.

14. Narayanan N, Johnson L, MacDougall C. Beyond Susceptible and Resistant, Part III: Treatment of Infections due to Gram-Negative Organisms Producing Carbapenemases. J Pediat Pharmacol Ther. [Internet]. 2016 [citado el 12 de noviembre del 2016]; 21(2):110-119. Disponible en: https://www. ncbi.nlm.nih.gov/pmc/articles/PMC486 9768/pdf/i1551-6776-21-2-110.pdf.

15. Leal AL, Cortes JA, Ovalle MV, Saavedra SY, Buitrago G, Escobar JA, et al. Emergencia de fenotipos resistentes a cefalosporinas de tercera generación en enterobacteriaceae causantes de infección del tracto urinario de inicio comunitario en hospitales de Colombia. Enferm Infecc Microbiol de Chile. [Internet]. 2013 [citado el 6 de mayo del 2016];31(5):298-303. Disponible en: https://medes.com/publication/81312.

16. Logan LK, Renschler JP, Gandra S, Weinstein RA, Laxminarayan R. Carbapenem-Resistant Enterobacteriaceae in Children, United States, 1999-2012. Emerg Infect Dis. [Internet] 2015 [citado el 7 noviembre del 2016]; 21(11):2014-2021. Disponible en: https://www.ncbi.nlm.nih.gov/pmc/arti cles/PMC4622248/pdf/15-0548.pdf.

17. Pannaraj PS, Bard JD, Cerini C, Weissman SJ. Pediatric Carbapenem-resistant Enterobacteriaceae in Los Angeles, California, a High-prevalence Region in the United States. Pediatr Infect Dis J. [Internet] 2015 [citado el 22 enero del 2016]; 34(1):11-16. Disponible en: https://www.ncbi.nlm.nih. gov/pmc/articles/PMC4437704/pdf/nih ms-680856.pdf.

18. Plan de Prevención y control frente a la infección por enterobacterias productoras de cabapenemasas (EPC) en la Comuni dad de Madrid. [internet]. Madrid: Secretaria de Salud de España; 2013. [citado el 15 
de mayo del 2016]. Disponible en: http:// docplayer.es/2965581-Plan-de-preven cion-y-control-frente-a-la-infeccion-por -enterobacterias-productoras-de-carbape nemasas-epc.html.

19. Lubbert C, Straube L, Shubert, Rodolfo A, Mosner, Sein C, et al. Colonization with extended spectrum beta lactamase producing and carbapenemase producing. Int $\mathrm{J}$ Med Microbiol. 2015 abril; 21(4): 148-156. Disponible en: https://www.ncbi.nlm.nih. gov/pubmed/25547265.

20. Lubbert C, Straube L, Stein C, Makarewicz O, Schubert S, Mossner J, et al. Colonization with extended-spectrum beta-lactamase-producing and carbapenemase-producing enterobacteriaceae in international travelers returning to Germany. Int J Med Microbiol. [Internet] 2015 [citado el 16 de noviembre del 2016]; 305(1):148-156. Disponible en: https://www.ncbi.nlm.nih. gov/pubmed/25547265.

21. Lefebvre $B$, Levesque $S$, Bourgalt $A M$, Mulvey M, Mataseje L, Boyd D, et al. Carbapenemen non susceptible enterobacteriaceae, Quebec Canada: results of a laboratory surveillance program 2010-2012. PLoS One. [Internet] 2015 [citado 30 de enero del 2016]; 10(4):e0125076. Disponible en: https:// www.ncbi.nlm.nih.gov/pmc/articles/ PMC4409364/pdf/pone.0125076.pdf.

22. Stein C, Makarewics O, Bohner JA, Pfeifer Y, Kesselmeier M, Hagel S, et al. Three dimensional checkerboard synergy analysis of colistin, meropenem, tigecycline against multidrug-resistant clinical klebsiella pneumonia isolates. PLoS One. [Internet] 2015 [citado 22 de enero del 2016]; 10(6):e0126479. Disponible en: http://journals.plos.org/plosone/article? id=10.1371/journal. . pone. 0126479 .

23. Cuervo SI, Sánchez R, Gómez-Rincón JC, Almenares C, Osorio JP, Vargas MJ. Comportamiento de casos de Klebsiella pneumoniae productora de carbapene masas en pacientes con cáncer de un hospital de tercer nivel de Bogotá, D.C. Biomédica. [Internet] 2014 [citado el 22 de Septiembre del 2016]; 34(1):170-180. Disponible en: http://www.revistabiomedi ca.org/index.php/biomedica/article/ view/1680.

24. Pollett S, Miller S, Hindler J, Uslan D, Carvalho $\mathrm{M}$, Uslan $\mathrm{D}$. Phenotypic and molecular characteristics of carbapenem resistant enterobacteriaceae in a health care system in Los Angeles from 2011 and 2013. J Clin Microbiol. [Internet] 2014 [citado el 21 noviembre del 2017; 52(11):4003-4009. Disponible en: https:// www.ncbi.nlm.nih.gov/pmc/articles/ PMC4313239/pdf/zjm4003.pdf.

25. Torres Gonzales $P$, Cervera Hernández ME, Niembro Ortega MD, Leal Vega F, Cruz-Hevert LP, García-García L, et al. Factors Associated to prevalence and incidence of carbapenem-resistant enterobacteriaceae fecal carriage: a cohort study in a mexican tertiary care Hospital. PLos One. [Internet]. 2015 [citado 28 de enero del 2016]; 10(10):e0139883.

Disponible en: https://www.ncbi.nlm.nih. gov/ pmc/articles/PMC4592225/pdf/pone. 0139883.pdf.

26. Biehle LR, Cottreau JM, Jessy C, Thompson DJ, Filipek RL, O'Donnell JN, et al. Outcomes and Risk Factors for Mortality among Patients Treated with Carbapenems for Klebsiella spp. Bacteriemia. PLoS One [Internet]. 2015 [citado 07 de enero del 2016]. Disponible en: https:// www.ncbi.nlm.nih.gov/pmc/articles/PM C4664260/pdf/pone.0143845.pdf.

27. Jamal WY, Albert MJ, Rotimi VO. High prevalence of New Delhi Metallo- $\beta$-lactamase-1 (NDM-1) producers among carbapenem-resistant enterobacteriaceae in kawait. PLoS One. [Internet] 2016 [citado el 31 de marzo del 2016]; 11(3): e0152638. Disponible en: https://www.nc bi.nlm.nih.gov/pmc/articles/PMC4816 385/pdf/pone.0152638.pdf. 